\title{
PIERROT'S THEOREM FOR SINGULAR RIEMANIAN FOLIATIONS
}

\author{
ROBERT A. WOLAK
}

\begin{abstract}
Let $\mathcal{F}$ be a singular Riemannian foliation on a compact connected Riemannian manifold $M$. We demonstrate that global foliated vector fields generate a distribution tangent to the strata defined by the closures of leaves of $\mathcal{F}$ and which, in each stratum, is transverse to these closures of leaves.
\end{abstract}

The aim of this short note is to prove M. Pierrot's theorem for singular Riemanian foliations, cf. [5], namely.

Theorem 1. Let $\mathcal{F}$ be an $S R F$ on a compact manifold $M$. Then the vector space of global foliated vector fields is transitive to the closures of leaves in each closure stratum.

\section{Preliminaries}

First we recall some and prove other results about SRF-s (singular Riemannian foliations), cf. [3] and [4].

Assume that the manifold $M$ is compact and connected (or the metric is complete). Then the closure of any leaf is a submanifold.

Let $k$ be any number between 0 and $n$. Define

$$
\Sigma_{k}=\left\{x \in M: x \in L_{\alpha}, \operatorname{dim} L_{\alpha}=k\right\} .
$$

The leaves of $\mathcal{F}$ is $\Sigma_{k}$ are of the same dimension, however they can have holonomy. P. Molino demonstrated that the sets $\Sigma_{k}$ or rather their connected components are submanifolds of $M$ and $\overline{\Sigma_{k}} \subset \bigcup_{i \leq k} \Sigma_{i}$. Note that for some $i$ the sets $\Sigma_{i}$ can be empty. Moreover, let $k_{0}$ be the maximum dimension of leaves of $\mathcal{F}$. Then the set $\Sigma_{k_{0}}$ is open and dense 
in $M$. It is the principal stratum. In fact, the partition $\left\{\Sigma_{k}\right\}_{0}^{n}$ is an abstract stratification.

Let $W$ be a compact submanifold of $M$. The geodesics define the exponential mapping exp : $N(W) \rightarrow M$. Denote by $S_{r}(W)=\{v \in$ $N(W):\|v\|=r\}$ (resp. $D_{r}(W)=\{v \in N(W):\|v\| \leq r\}$ ) and by $S(W, r)$ (resp. $D(W, r))$ its image by exp. If $W$ is a closed leaf or the closure of a stratum then it is not difficult to notice that leaves of the foliation $\mathcal{F}$ live on $S(W, r)$, cf. [3], [4]. Moreover, the homotethies (along the geodesics) $h_{\lambda}: D(W, r) \rightarrow D(W,|\lambda| r), h_{\lambda}(\exp (v))=\exp (\lambda v)$ preserve the foliation. The leaf passing through $\exp (v)$ has the same dimension and holonomy as the leaf passing through $\exp (\lambda v)$.

Connected components of $\Sigma_{i}$ are submanifolds of $M$. They can be of different codimension and it can happen that some connected component of $\Sigma_{i}$ is a compact submanifold. Since the foliation is Riemannian the closure of a leaf from a stratum $\Sigma_{i}$ remains in it. In fact, let $\partial \Sigma_{i}=V_{1} \cup$ $\cdots \cup V_{k}$. where $\bigcup_{s=1}^{k} V_{s}=\bar{\Sigma}_{i}-\Sigma_{i}$, each $V_{s}$ being a connected submanifold of $M$. In a tubular neighbourhood of $V_{s}$ leaves of $\mathcal{F}$ live on the sphere bundles $S\left(V_{s}, r\right)$. Thus if $L \subset S\left(V_{s}, r\right)$, so does its closure $\bar{L}$. Therefore for all our purposes the foliation $\mathcal{F} \mid \Sigma_{i}$ behaves like a $\mathrm{RF}$ on a compact manifold. Therefore we can define the subspaces

$$
\Sigma_{i j}=\left\{x \in \Sigma_{i}: x \in L \in \mathcal{F}, \operatorname{dim} \bar{L}=j\right\} .
$$

Each $\Sigma_{i j}$ is a submanifold of $\Sigma_{i}$ and $\partial \Sigma_{i j_{0}} \subset \bigcup_{s<i} \Sigma_{s} \bigcup_{j<j_{0}} \Sigma_{i j}$. The closures of leaves of $\mathcal{F}$ induce a regular RF $\mathcal{F}_{i j}$ of compact leaves on $\Sigma_{i j}$. The leaves of $\mathcal{F}_{i j}$ have finite holonomy. Using the exponential mapping restricted to the normal bundle of a leaf one easily learns that the holonomy of a leaf is conjugated to the linear holonomy of this leaf. The linear holonomy is a finite subgroup of the linear orthogonal group. The linear holonomy groups $h(L, x)$ at different points $x$ of a given leaf $L$ are conjugated; let us denote this conjugacy class by $h(L)$. If $\alpha$ denotes a conjugacy class of a subgroup of the linear orthogonal group then let $\Sigma_{i j \alpha}=\left\{x \in \Sigma_{i j}: x \in L \in \mathcal{F}_{i j}, h(L)=\alpha\right\}$.

In [5] M. Pierrot uses a slightly rougher stratification for regular RFs, namely

$$
\Sigma_{p j k}=\{x \in L \in \mathcal{F}: \operatorname{dim} \bar{L}=j, \sharp h(\bar{L}, x)=k\}
$$

where $p=\operatorname{dim} \mathcal{F}$, and the holonomy is considered in the stratum $\Sigma_{j}$. However, in a tubular neighbourhood of a compact leaf $\bar{L}$, the foliation $\overline{\mathcal{F}}$ by the closures of leaves, is conjugated to the natural foliation of the flat bundle $\bar{L} \times{ }_{G} R^{s}$ where $G$ is the linear holonomy group of the leaf $\bar{L}$ and $s=\operatorname{codim}_{\Sigma_{j}} \bar{L}$. It is not difficult to notice that in these tubular neighbourhoods leaves of $\overline{\mathcal{F}}$ have their linear holonomy groups conjugated to 
a subgroup of $G$. It means that for any $\alpha, G \in \alpha, \sharp G=k \Sigma_{p j \alpha} \subset \Sigma_{p j k}$ and the submanifolds $\Sigma_{p j k}$ are separated. If $\Sigma_{p j \alpha}$ and $\Sigma_{p j \beta}$ are two such sets then the lemma concerning the homotethies, cf. [3], [4], ensures that $\overline{\Sigma_{p j \alpha}} \cap \overline{\Sigma_{p j \beta}}=\emptyset$. Therefore connected components of $\Sigma_{p j \alpha}$ are also connected components of $\Sigma_{p j k}$. Thus connected components of these sets define the same stratification $\left\{\Sigma_{\gamma}\right\}$. The stratification $\left\{\Sigma_{\gamma}\right\}$ possesses a natural partial order

$$
\Sigma_{\gamma} \leq \Sigma_{\gamma^{\prime}} \text { iff } \Sigma_{\gamma} \subset \bar{\Sigma}_{\gamma^{\prime}}
$$

The strata defined above we call the closure strata of the foliation $\mathcal{F}$ to distinguish them from the strata defined by the dimension of leaves.

In [3], [4] P. Molino describes a way of desingularization of SRFs. Let $\Sigma$ be a minimal stratum. $\Sigma$ is a closed submanifold. Let $N(\Sigma)$ be the normal bundle of $\Sigma$. Leaves of $\mathcal{F}$ also live on sphere bundles $S(\Sigma, r)$ over $\Sigma$. Take $M^{0}=(M-\Sigma) \times\{0\}, M^{1}=(M-\Sigma) \times\{1\}$ and $S=S(\Sigma, r) \times(-1,1)$ for some $r>0$. Then $M^{0}, M^{1}$ and $S$ glue together to become a compact manifold $M_{1}$, i.e. $S(\Sigma, r) \times\{t\}$ is identified with $S(\Sigma,|t| r) \times\{0\} \subset M^{0}$ if $t<0$ and with $S(\Sigma,|t| r) \times\{1\} \subset M^{1}$ if $t>0$. $M_{1}$ projects onto $M, p: M_{1} \rightarrow M$. Over $M-\Sigma p$ is a double covering and $p^{-1}(\Sigma)=S(\Sigma, r)$.

P. Molino proves that on $M_{1}$ there exists an SRF $\mathcal{F}_{1}$, which does not have leaves of the type encountered in $\Sigma$, and including the old foliation $\mathcal{F}$ on $M^{0}$ and $M^{1}$. After a finite number of steps we get a regular Riemannian foliation on a compact manifold $M_{s}$.

Using the exponential mapping it is quite easy to prove a following lemma.

Lemma 1. For any $0<\delta_{1}<\delta_{2} \leq \epsilon$ there exists a basic smooth function

$$
\lambda\left(\delta_{1}, \delta_{2}\right): D(\Sigma, \epsilon) \rightarrow[0,1]
$$

such that $\operatorname{supp} \lambda\left(\delta_{1}, \delta_{2}\right) \subset D\left(\Sigma, \delta_{2}\right)$ and $\lambda\left(\delta_{1}, \delta_{2}\right) \mid D\left(\Sigma, \delta_{1}\right) \equiv 1$.

In our future considerations we shall need the following relations between basic functions on the foliated manifolds $(M, \mathcal{F})$ and $\left(M_{1}, \mathcal{F}_{1}\right)$.

Lemma 2. Let $f$ be a basic function on $\left(M_{1}, \mathcal{F}_{1}\right)$. Then for any point $x \in M^{0}$ there exists a foliated neighbourhood $U$ of $x$ in $M^{0}$ and a basic function $f_{U}$ on $(M, \mathcal{F})$ such that $f_{U} p|U=f| U$.

Proof: The set $D(\Sigma, \epsilon)-\Sigma=D^{0}(\Sigma, \epsilon)$ can be considered as (via $p$ ) an open subset of $M^{0}$. Therefore we have to consider two cases: (a) $x \notin D^{0}(\Sigma, \epsilon)$ and (b) $x \in D^{0}(\Sigma, \epsilon)$. 
In the case (a) as $U$ we can take $M-D\left(\Sigma, \delta_{2}\right), 0<\delta_{2}<\epsilon$ and as $f_{U}$ the function

$$
\begin{cases}f(z) & z \notin D^{0}(\Sigma, \epsilon) \\ \left(1-\lambda\left(\delta_{1}, \delta_{2}\right)\right) f(z) & z \in D^{0}(\Sigma, \epsilon), 0<\delta_{1}<\delta_{2} \\ f(z)=0 & z \in \Sigma .\end{cases}
$$

In the case (b) let $x \in S(\Sigma, r), 0<r \leq \epsilon$. Then we take $U=$ $M-D(\Sigma, r / 2)$ and define the function as in the case (a) taking $0<\delta_{1}<$ $\delta_{2}<r / 2$.

Lemma 3. Let $f$ be a basic function on the foliated manifold $(M, \mathcal{F})$. Then for any point $x$ of $M-\Sigma$ there exists an open foliated neighbourhood $U$ of $x$ in $M-\Sigma$ and a basic function $f_{U}$ on $\left(M_{1}, \mathcal{F}_{1}\right)$ such that $f \mid U p=$ $f_{U} \mid M^{0} \cap p^{-1}(U)$.

Proof: It is analogous to that of Lemma 2. Using this construction we obtain a basic function $\hat{f}_{U}$ with compact support on $\left(M^{0}, \mathcal{F}_{1}\right)$; we extend it to $M_{1}$ putting 0 on $\Sigma$ and $M^{1}$.

Let us recall the definition of the 'musical' isomorphism, for example cf. $[\mathbf{1}]$.

$$
\text { b : } T M \rightarrow T^{*} M
$$

is given by: for $X \in T M_{x} X^{b}$ is the only 1-form such that

$$
\begin{gathered}
g(X, Y)=X^{b}(Y) \text { for any } Y \in T M_{x} . \\
\sharp: T^{*} M \rightarrow T M
\end{gathered}
$$

for any $\omega \in T^{*} M_{x} \omega^{\sharp}$ is the only vector for such that

$$
g\left(\omega^{\sharp}, Y\right)=\omega(Y) \text { for any } Y \in T M_{x} .
$$

Therefore to any function $f$ on $M$ we associate a vector field $X^{f}$ by the formula

$$
g\left(X^{f}, Y\right)=d f(Y) \text { for any } Y \in T M \text { or } X^{f}(x)=\left(d f_{x}\right)^{\sharp} .
$$

Now we shall study the properties of vector fields associated to basic functions. First let us notice that for any basic function $f$ the vector field $X^{f}$ is orthogonal to the leaves of the foliation. Moreover if the function $f$ is global the vector field $X^{f}$ is orthogonal to the closures of leaves.

Lemma 4. If $f$ is a basic function then the vector field $X^{f}$ is an infinitesimal automorphism of the foliation.

The proof is a straightforward calculation. 


\section{Regular case}

Let $\mathcal{F}$ be an RF. We shall look at the existence of global basic functions. Denote $\mathcal{X}^{\sharp}(M, \mathcal{F})$ the vector space of global vector fields of the form $X^{f}$ for some global basic function $f$ on $(M, \mathcal{F})$.

The closures of leaves form an SRF and we can consider strata for this foliation, cf. [5]. These strata are just our closure strata for $\mathcal{F}$ as $\mathcal{F}$ being regular we have just the principal stratum for this foliation. It is obvious that global infinitesimal automorphisms must be tangent to the closure strata. Let $\Sigma$ be one of these strata.

Lemma 5. For any vector $X \in T \Sigma_{x}$ orthogonal to the closure $S$ of the leaf $L$ in $\Sigma$ passing through $x$, there exists a global basic function $f$ such that $d f(X) \neq 0$.

Proof: There exists $\epsilon>0$ such that the mapping $\exp _{S}: B_{\epsilon}(X) \rightarrow M$ is an embedding. Then there is a leaf $L^{\prime}$, with the closure $S^{\prime}$, of the same stratum $\Sigma$ on the geodesic with the initial condition $X$ at the distance less than $\epsilon$ such that the mapping $\exp _{S^{\prime}}: B_{\epsilon}\left(S^{\prime}\right) \rightarrow M$ is an embedding, cf. [2]. Then the function $f_{S^{\prime}}(y)=d\left(y, S^{\prime}\right)^{2}$ is a smooth basic function on $\exp _{S^{\prime}}\left(B_{\epsilon}\left(S^{\prime}\right)\right)$ for which $d f_{S^{\prime}}(X) \neq 0 . f_{S^{\prime}}$ can be easily extended to a global basic function.

Combining Lemmas 4 and 5 we get the following proposition which, in fact, is a variant of the theorem due to M. Pierrot, cf. [5].

Proposition 1. Let $(M, \mathcal{F})$ be a compact foliated manifold with $\mathcal{F}$ being a regular $R F$. Then the vector space $\mathcal{X}^{\sharp}(M, \mathcal{F})$ is transitive to the closures of leaves in each closure stratum.

\section{Singular case}

Now let $\mathcal{F}$ be an SRF on $M$. First we prove the singular version of Lemma 5.

Lemma 6. Let $(M, \mathcal{F})$ be a compact foliated manifold with $\mathcal{F}$ being an SRF. Let $\Sigma$ be a closure stratum of $\mathcal{F}$. For any vector $X \in T \Sigma_{x}$ orthogonal to the closure $S$ of the leaf $L$ passing through $x$ there exists a basic function $f$ such that $d f(X) \neq 0$.

Proof: Using the blowing up procedure and Lemma 2 we can reduce our considerations to the case where the point $x$ belongs to the singular stratum $\Sigma_{0}$ of the foliation $\mathcal{F}$. Thus $\Sigma$ is a submanifold of $\Sigma_{0}$ and a 
closure stratum of $\left(\Sigma_{0}, \mathcal{F}\right)$ which is compact RM. Therefore according to Lemma 5 there exists a basic function $f_{0}$ on $\Sigma_{0}$ such that $d f_{0}(X) \neq 0$. According to the next lemma this basic function can be easily extended to a global basic function on $(M, \mathcal{F})$.

Lemma 7. Any basic function on a stratum $\Sigma$ can be extended to a global basic function on $M$.

Proof: Since the projection $p: B(\Sigma, \epsilon) \rightarrow \Sigma$ maps leaves onto leaves, for any basic function $f$ on $\Sigma$, the function $f p$ is basic on $B(\Sigma, \epsilon)$. Then using a function $\lambda\left(\delta_{1}, \delta_{2}\right)$ we can extend $f p$ to a global basic function on $(M, \mathcal{F})$.

For vectors which are not tangent to strata we have the following lemma.

Lemma 8. Let $\mathcal{F}$ be an $S R F$ on a compact manifold $M$. If a vector field $X$ is not tangent to the closure of a leaf $L$ at a point $x$, then there exists a global basic function $f$ such that the germ at $x$ of the function $d f(X)$ is not 0.

Proof: Let $S$ be the closure of the leaf $L$. It is a compact submanifold of $M$. Let $N(S)$ be its normal bundle. For some $\epsilon>0$ the exponential mapping defined by the geodesics starting from vectors of $N(S)$ is a diffeomorphisms of $B_{\epsilon}(S)=\{v \in N(S):\|v\|<\epsilon\}$ onto the image $B(S, \epsilon)$, cf. [4]. Using a similar method as in Lemma 2 we can extend any basic function on $B(S, \epsilon)$ to a global one. Therefore we have reduced our problem to a local one. Then the function

$$
f_{L}(y)=d(L, y)^{2}
$$

satisfies the conditions of the lemma.

\section{Proof of Theorem 1}

Let $x$ be any point of a closure stratum $\Sigma$. Let $V$ be the subspace of $T_{x} \Sigma$ orthogonal to $T_{x} S, S=\bar{L}_{x}$. We know that for any global basic function $f X_{x}^{f} \in V$. Lemma 6 ensures that there does not exist a vector in $V$ which is orthogonal to all $X_{x}^{f}$. It means precisely that $S P A N\left\{X_{x}^{f}\right\}=$ $V$. Therefore we have proved the following theorem:

Theorem 2. Let $M$ be a compact connected manifold and $\mathcal{F}$ be an $S R F$ on $M$. Then the vector space $X^{\sharp}(M, \mathcal{F})$ is transitive to the closures of leaves in each closure stratum of $(M, \mathcal{F})$.

Of course Theorem 2 is just a more detailed version of Theorem 1 . 


\title{
References
}

1. Gallot S., Hulin D. and Lafontaine J., "Riemannian Geometry," Springer, 1987.

2. Hirsch M., "Differential Topology," Springer, 1976.

3. Molino P., "Riemannian Foliations," Progress in Math. 73, Birkhäuser, 1988.

4. Molino P., "Feuilletages riemanniens reguliers et singuliers, Géometrie et Physique," Paris, 1986.

5. Pierrot M., Orbites des champs feuilletés pour un feuilletages riemanniens sur une variété compacte, C.R. Acad. Sc. Paris 301 (1985), 443-445.

\author{
Instytut Matematyki \\ Uniwersytet Jagiellonski \\ Wl. Reymonta 4 \\ 30-059 Krakow \\ POLAND
}

Rebut el 29 d'Abril de 1994 